\title{
A novel SETI strategy targeting the solar focal regions of the most nearby stars
}

\author{
Michaël Gillon \\ Institut d'Astrophysique et de Géophysique, Université de Liège, Allée du 6 Août 17, \\ Bât. B5C, 4000 Liège, Belgium
}

\begin{abstract}
Many hypotheses have been raised to explain the famous Fermi paradox. One of them is that self-replicating probes could have explored the whole Galaxy, including our Solar System, and that they are still to be detected. In this scenario, it is proposed here that probes from neighboring stellar systems could use the stars they orbit as gravitational lenses to communicate efficiently with each other. Under this hypothesis, a novel SETI approach would be to monitor the solar focal regions of the most nearby stars to search for communication devices. The envisioned devices are probably not detectable by imagery or stellar occultation, but an intensive multi-spectral monitoring campaign could possibly detect some communication leakages. Another and more direct option would be to message the focal regions of nearby stars in an attempt to initiate a reaction.
\end{abstract}

Keywords: Astrobiology, Extraterrestrial intelligence, Interstellar communication, Interstellar probes

\section{Introduction}

In 1959, two scientists proposed to search for interstellar communications from extrasolar civilizations [1]. The first application of this idea was performed a few months later [2], effectively inaugurating the modern era of SETI, the Search for Extra-Terrestrial Intelligence. Since then, a large number of SETI projects have been conducted, most of them focusing on the

Email address: michael.gillon@ulg.ac.be (Michaël Gillon) 
search for radio emissions from advanced civilizations trying to communicate with us [3].

Despite the fact that no confirmed extraterrestrial signal has been detected so far, many discoveries obtained within the last fifty years have strongly reinforced the scientific rationale of the SETI enterprise. First, we now know that planets are the normal by-products of stellar formation, and that most stars of our Galaxy harbor planets, including terrestrial planets similar to Earth and orbiting in the so-called circumstellar habitable zone [4, 5, 6]. Second, there is now strong evidence that life appeared on Earth a few hundred millions years at most after our planet had cooled enough to support liquid water on its surface [7]. This early emergence is consistent with a high probability of abiogenesis providing a few conditions are met, mostly liquid water on the surface of an organics-rich terrestrial planet (or satellite) with a stable source of energy [8]. Third, biologists have discovered life forms surviving in some of the harshest environments of Earth. The majority of these so-called extremophiles are microbes (Eubacteria and Archaea), but some multicellular animal examples are also known (e.g. Tardigrades, Antarctic krills). Extremophiles have revolutionized our vision of the adaptability of life, significantly expanding the plausible limits for life on other planets [9].

Taken as a whole, these results are consistent with the ubiquitous presence of life in the Galaxy. Furthermore, many examples of evolutionary convergence known on Earth indicate that our high level of intelligence and its resulting technology could be not an extraordinary stroke of luck, but one among the finite number of outcomes to the natural selection mechanisms that should be in work in any biosphere [10, 11, 12]. All these considerations make the emergence of other civilizations in the Galaxy since its birth more than 10 Gy ago a plausible hypothesis that deserves to be comprehensively addressed.

Our own technological civilization is less than two hundred years old, and we have already sent robotic probes to a large number of bodies of our Solar System. Our technology is certainly not yet mature enough to build a probe able to reach one of the nearest stars in a decent time (i.e. within a few decades), but nothing in our physical theories precludes such a project. On the contrary, the constant progress in the fields of space exploration, nanotechnology, robotics and electronics, combined with the development of new possible energy sources like fusion reactors or solar sails, indicate that interstellar exploration could become a technological possibility in the future, 
provided that our civilization persists long enough. So, if extrasolar technological civilizations have existed in the Milky Way, we cannot discard the intriguing possibility that at least one of them has visited our Solar System. The plausibility of this hypothesis is strongly increased when considering the concept of self-replicating interstellar probes [13, 14], i.e. probes that would be sent by a civilization to a few neighboring planetary systems where they would mine raw materials to create replicas of themselves that would head towards other nearby systems, replicate there, and so on. With such a strategy very similar to the growth of a bacterial colony, it has been shown that a single self-replicating probe based on conventional physics and existing technological concepts could spread throughout the Galaxy within a few hundreds of millions years at most [14, 15].

The absence of such 'Von Neumann probes' in our Solar System has been presented as a strong argument against the existence of extraterrestrial civilizations [14]. This new formulation of the Fermi paradox [16] assumes that an extraterrestrial probe monitoring the Solar System would be easy to detect. This is far from the truth. From the Sun to the external limit of the Oort cloud, the Solar System is a $5 \times 10^{14} \mathrm{AU}^{3}$ volume that remains mostly unexplored [17, 18]. Several unsuccessful attempts have been made to detect extraterrestrial artifacts in the Solar System (e.g. [19, 20, 21]), but they have probed only a tiny fraction of the Solar System, and concluding at this point that our Solar System does not contain any extraterrestrial probe is definitely premature. Here we present a new strategy for testing the existence of such probes, based on a hypothesis presented in the next section.

\section{Proposed hypothesis}

The concept of Von Neumann probes exploring the Galaxy requires some level of communication and coordination between the probes. One could hypothesize that the probes would directly communicate with their original system, but this hypothesis is a priori very unlikely. The vastness and structure of the Milky Way makes impossible (at least for our technology) a direct communication between a large fraction of stars, for instance between the Sun and a star located at the opposite side of the galaxy and hidden by the galactic center. Furthermore, the coordination of probes to explore neighboring systems would be very inefficient, considering the limited speed of light and the large distances in play. A communication strategy based on direct connexions between neighboring systems would be a much bet- 
ter solution, with the extra-benefit that the information gathered by probes would be spread among their whole network, without any loss even in case of collapse or migration of the original civilization. The first part of our hypothesis is thus that the envisioned probes would use this direct communication strategy.

The second part of our hypothesis concerns the technique used by the probes for their interstellar communication. In this respect, every star represents a huge advantage, thanks to its large mass that, according to Einstein's theory of general relativity [22], deflects the light rays passing close to it. This effect, observed for the first time for the Sun in 1919 [23], makes any star a potential gravitational lens. As first outlined by Von Eshleman in 1979 [24], using the Sun as a gravitational lens would provide an unprecedented potential for astronomical observations and interstellar communication. This potential was further studied by Maccone [25, 26, 27, 28] who confirmed the tremendous advantage of the effect for efficient interstellar communication. For instance, at $32 \mathrm{GHz}$, the combined transmission gain brought by the Sun and Alpha Cen $\mathrm{A}$ is $\sim 10^{16}$, making possible an efficient communication between the two stars with very moderate transmission powers. In fact, Maccone's most striking conclusion is that it is only by using the gravitational focusing potential of the Sun that we would be able to communicate with a probe sent to any of the nearest stars [29]. Basing on this conclusion, it is assumed here that Von Neumann probes would use the same technique. This is the second part of our hypothesis.

\section{Testing the hypothesis}

Assuming the colonization of the whole Galaxy by self-replicating probes, our working hypothesis presented above predicts that we should find an interstellar communication devices (ICD) at the solar focus of at least one nearby star. This 'focus' is in fact not localized, but any point above a specific distance on a line connecting the star to the Sun, in the opposite direction to the star. Using the Schwarzschild metric to represent the gravitational field around the Sun, one can compute a value of 550 AU for this minimum distance (e.g. [28]). In practice, a larger distance is required, especially at lower frequencies, because of the scattering properties of the solar corona. A typical distance of $1000 \mathrm{AU}$ is probably a better estimation [28].

For illustrative purpose, we consider from now on Alpha Centauri as the target system. From Earth, the position of the putative ICD relative to the 
background stars would not be fixed but would show a large amplitude (a few arcmin) 1yr-period elliptical movement (in first approximation) due to the annual parallax (Fig. 1). This corresponds to a mean offset of 5" per day. In addition, the ICD would show a motion in opposite direction to the proper motion of Alpha Cen (3.6" /yr in RA and 0.7"/yr in DEC).

To assess the potential for detecting the envisioned ICS, some working assumptions are now made about it, based on our current or foreseen technology. For the antenna itself, a diameter of one or two dozens of meters at most can be assumed, based on the design of FOCAL, a proposed space mission that would use the Sun as a gravitational lens [25, 26, 27, 28, 29, 30]. That is the whole point of using a star as lens for interstellar communication: relatively small antennae and emission powers are required. For the mass of the payload, it is assumed to be not much larger than 1 ton. As a comparison, the mass of the Voyager 1 spacecraft is $722 \mathrm{~kg}$. By keeping the same azimuthal position in a barycentric inertial system, the ICD would drift radially towards the Sun. To compensate for the gravitational pull of the Sun, a solar sail solution seems promising as it requires no intrinsic energy source, so it is adopted here for our ICD toy model. A circular sail facing the Sun is assumed, adopting as effective radiation pressure at $1 \mathrm{AU} P_{\text {eff }}=9.1 \mu \mathrm{N} / \mathrm{m}^{2}$, corresponding to an ideal sail (perfectly flat, 100\% specular reflection) [31]. For the sail surface density $\rho_{s}$, an upper limit is imposed from the following equation that equals the gravity and radiation forces:

$$
\frac{G M_{*}\left(M_{p}+S_{s} \rho_{s}\right)}{r^{2}}=P_{e f f} S_{s}
$$

where $G$ is the gravity constant, $M_{*}$ and $M_{p}$ are respectively, the mass of the Sun and of the payload, $S_{s}$ is the surface of the sail, and $r=1 \mathrm{AU}$. Rearranging to solve for the surface of the sail leads to

$$
S_{s}=\frac{G M_{*} M_{p}}{r^{2} P_{e f f}-G M_{*} \rho_{s}}
$$

With $P_{\text {eff }}=9.1 \mu \mathrm{N} / \mathrm{m}^{2}$, this equation is physically valid only for $\rho_{s}<1.4$ $\mathrm{g} / \mathrm{m}^{2}$, making this value an absolute upper limit for the sail surface density. Solar sail material of such low density has not yet been manufactured on an industrial scale, but still prototypes composed of nanotubes and with densities down to $0.1 \mathrm{~g} / \mathrm{m}^{2}$ do exist [32]. For the purpose of this work, a value of $0.5 \mathrm{~g} / \mathrm{m}^{2}$ is adopted. This results into a circular sail of $\sim 550$ meters radius and of $\sim 0.5$ ton mass. 
Figure 2 shows the visual magnitude of the sail at zero phase angle as a function of the payload mass, assuming a Bond albedo of 1 and neglecting light scattering. For a 1 ton payload, the visual magnitude would be $\sim 30.5$. This is in fact an absolute lower limit, as for an actual sail, the reflection would not be purely specular. Furthermore, assuming a zero phase angle means that the telescope would have to be on the line connecting the ICD to the Sun, i.e. it would have to transit the Sun as seen from the ICD. For an Earth-bound telescope and an ICD targeting Alpha Cen, this is not possible, as Alpha Cen is not in the ecliptic plane (ecliptic latitude $=43^{\circ}$ ). Even assuming one could observe the ICD sail at zero phase angle, the use of the exposure time calculator simulator for the future E-ELT $39 \mathrm{~m}$ telescope [33, 34] results in a cumulative exposure time of several weeks to detect a 30.5-magnitude point-source with a decent signal-to-noise ratio of 10 . This means that detecting the envisioned ICD using direct imaging techniques is not feasible with existing or upcoming facilities.

The stellar occultation technique has been presented as a promising method for detecting small objects of the outer Solar System [35]. Using the formalism presented in [36], we estimated the parameters of the occultation pattern for a faint and distant background star occulted by the envisioned ICD, assuming observations at $600 \mathrm{~nm}$. The angular size of the sail at $1000 \mathrm{AU}$ would be $\sim 1.5 \mu$ arcsec, making the occultation pattern completely dominated by diffraction. The angular radius of the occulted star was assumed to be 20 $\mu$ arcsec, corresponding to a $\sim 2 R_{\odot}$ star at 500 pc. This assumption does not alter the conclusions. The result is an occultation of $\sim 7 \%$ depth at most (for a null impact parameter) and $\sim 1$ s duration. Detecting such a short and shallow photometric event on a (most probably) very faint star would be a real challenge with existing or planned facilities. Furthermore, the expected number of occultations per year is only $3.5 \times 10^{-4}$, basing on the field density of stars brighter than magnitude 21 estimated from the GSC-II and USNO catalogues, and on the sky area covered by the ICD during its parallactic ellipse. Thanks to the proper motion of Alpha Cen, the sail would probe a slightly different area of the sky each year, nevertheless the expected time between two occultations is of the order of several thousand years, leading to the conclusion that the occultation technique is not a suitable method of detection either.

The two remote observational techniques commonly used to detect faint and small objects in the Solar System should thus be unable to detect the envisioned ICD. Another option is of course in situ exploration. In case of 
realization of a FOCAL-like space mission targeting Alpha Cen or another nearby star in the future, it would be interesting to equip the spacecraft with instruments able to explore efficiently the focal region in search of the envisioned ICD, not only efficient imaging devices but also powerful radar and/or lidar systems.

Attempts to detect the hypothesized ICD can still be performed now, basing on the very purpose of the device: not only to receive messages from Alpha Cen, but also to send messages to Alpha Cen and to one or several probes orbiting the Sun. An intense multi-spectral monitoring of the focal region of Alpha Cen with, e.g., the Allen Telescope Array [37], could in principle detect some leakages in these communications, depending on the used technology, communication frequency, and emission power.

Last but not least, a more direct detection strategy exists. Under the presented hypothesis, the astronomical position of the ICD is very well known, so we could simply attempt to message it, with the hope to cause its reaction (either by responding back, or via an increased chatter with other probes). Such an 'active SETI' [38] strategy has been criticized by several scientists basing on the risk of revealing our location to an hostile expansionary civilization (e.g. [39], [40]). In the present case, such criticisms would not apply, as it is difficult to imagine how extraterrestrial robotic probes having monitored our Solar System for probably a very long time could not already know that Earth does host life, including our nascent civilization.

\section{Conclusion}

It was hypothesized here that our Galaxy has been colonized by selfreproducing probes and that probes in neighboring systems communicate regularly using the stars they orbit as gravitational lenses for their interstellar communication. Under this hypothesis, an ICD should be present in the Solar System, in the focal region of (at least) one nearby star, at $\sim 1000 \mathrm{AU}$ for the Sun. It was argued that the traditional techniques of optical imaging and stellar occultation would probably not be able to detect the envisioned ICD, and that our best chances of detection in the near future relies on the intense multi-spectral monitoring of these regions, with the hope to catch a communication leakage, and on directly messaging the focal regions in attempts to initiate a reaction of the envisioned probes. While negative results could be explained not only by the non-existence of the envisioned ICD but also by a stealth and discretion policy of the hypothesized probes, a 
positive result would definitely revolutionize our understanding of our place in the Universe.

\section{Acknowledgments}

The author is a Research Associate of the Fonds National de la Recherche Scientifique-FNRS., and thanks B.-O. Demory, L. Delrez, G. Harp, E. Jehin, C. Maccone, P. Magain, J. C. Tarter, A. H. M. J. Triaud \& A. Zaitsev for useful comments and suggestions.

\section{References}

[1] G. Cocconi, P. Morrison, Searching for interstellar communications, Nature 184 (1959) 844846.

[2] F. D. Drake, Project Ozma, Phys. Today 14 (1961) 40-46.

[3] J. Tarter, The Search for Extraterrestrial Intelligence (SETI), Ann. Rev. Astron. Astrophys. 39 (2001) 511-548.

[4] A. Cassan, D. Kubas, J.-P. Beaulieu, el al., One or more bound planets per Milky Way star from microlensing observations, Nature 481 (7389) (2012) 167-169.

[5] C. D. Dressing, D. Charbonneau, The occurrence rate of small planets around small stars, Astrophys. J. 767 (1) (2013) 1-20.

[6] H. A. Howard, Observed properties of extrasolar planets, Science 340 (6132) (2013) 572-576.

[7] C. F. Chyba, K. P. Hand, Astrobiology: The Study of the Living Universe, Ann. Rev. Astron. Astrophys. 43 (2005) 31-74.

[8] C. H. Lineweaver \& T. M. Davis, Does the rapid appearance of life on Earth suggest that life is common in the universe? Astrobiol. 2 (2002) 293-304.

[9] R. Cavicchioli, Extremophiles and the Search for Extraterrestrial life, Astrobiol. 2 (3) (2002) 281-292. 
[10] C. de Duve, Vital Dust: life as cosmic imperative, New York, Basic Books (1995).

[11] N. J. Emery, N. S. Clayton, The Mentality of Crows: Convergent Evolution of Intelligence in Corvids and Apes, Science 10 (306) (2004) 19031907.

[12] F. B. M. de Waal, P. F. Ferrari, Towards a bottom-up perspective on animal and human cognition, Trends in Cognitive Science 14 (5) (2010) 201-207.

[13] R. A. Freitas, A self-reproducing interstellar probe, J. Br. Interplanet. Soc. 33 (1980) 251-264.

[14] F. J. Tipler, Extraterrestrial Intelligent Beings do not Exist, Q. J. R. Astron. Soc 21 (1980) 267-281.

[15] F. Valdes \& R. A. Freitas, Comparison of reproducing and nonreproducing starprobe strategies for galactic exploration, J. Br. Interplanet. Soc. 33 (1980) 402-406.

[16] C. Sagan, Direct contact among galactic civilizations by relativistic interstellar spaceflight, Planet. Space Sci. 11 (5) (1963) 485-498.

[17] R. Burke-Ward, Possible existence of extra-terrestrial technology in the solar system, J. Br. Interplanet. Soc. 53 (2000) 2-12.

[18] J. Haqq-Misra \& R. K. Kopparapu, On the likelihood of non-terrestrial artifacts in the Solar System, Acta Astronaut. 72 (2012) 15-20.

[19] M. D. Papagiannis, Are we alone, or could they be in the asteroid belt, Q. J. R. Astron. Soc. 19 (1978) 277-281.

[20] R. A. Freitas, A search for natural or artificial objects located at the earth-moon libration points, Icarus 42 (1980) 442-447.

[21] F. Valdes, R. A. Freitas, A search for objects near the earth-moon Lagrangian points, Icarus 53 (1983) 453-457.

[22] A. Einstein, Die Feldgleichungen der Gravitation, Sitzungsberichte der Preussischen Akademie der Wissenschaften zu Berlin (1915) 844847. 
[23] F. W. Dyson, A. S. Eddington, C. R. Davidson, A Determination of the Deflection of Light by the Sun's Gravitational Field, from Observations Made at the Solar Eclipse of May 29, 1919, Phil. Trans. Roy. Soc. 220 (571-581) (1920) 291-333.

[24] R. von Eshleman, Gravitational Lens of the Sun: Its Potential for Observations and Communications over Interstellar Distances, Science 205 (1979) 1133-1135.

[25] C. Maccone, Space missions outside the solar system to exploit the gravitational lens of the Sun, J. Br. Interplanet. Soc. 47 (1994), 45-52.

[26] C. Maccone, M. Piantà, Magnifying the nearby stellar systems by "FOCAL" space missions to 550 AU. Part I. J. Br. Interplanet. Soc. 50 (7) (1997) 277-280.

[27] C. Maccone, The gravitational lenses of alpha centauri a, b, c and of Barnard's star, Acta Astronaut. 47 (12) (2000) 885-897.

[28] C. Maccone, Deep space flight and communications - exploiting the Sun as a gravitational lens, ISBN978-3-540-72942-6 Springer-Verlag Berlin Heidelberg, NewYork, (2009).

[29] C. Maccone, Focusing the Galactic Internet, in Searching for Extraterrestrial Intelligence, The Frontiers Collection, ISBN 978-3-642-13195-0, Springer-Verlag Berlin Heidelberg (2011) 325-349.

[30] C. Maccone, Sun Focus Comes First, Interstellar Comes Second, J. Br. Interplanet. Soc. 66 (2013) 25-37.

[31] J. Wright, Space Sailing, ISBN 2-88124-803-9, Gordon and Breach Science Publishers (1992).

[32] M. Zhang, S. Fang, A. A. Zakhidov, et al., Strong, Transparent Multifunctional, Carbon Nanotube Sheets, Science 309 (5738) (2005) 12151219 .

[33] A. McPherson, R. Gilmozzi, J. Spyromilio, M. Kissler-Patig, S. Ramsay, et al., Recent Progress towards the European Extremely Large Telescope (E-ELT), The Messenger 148 (2012) 1-8. 
[34] http://www.eso.org/observing/etc/doc/elt/helpelt.html

[35] M. Bailey, Can 'invisible' bodies be observed in the solar system? Nature 259 (1976) 290-291.

[36] C. Nihei, M. J. Lehne, F. B. Bianco, et al., Detectability of Occultations of Stars by Objects in the Kuiper Belt and Oort Cloud, The Astronomical Journal 134 (2007) 1596-1612.

[37] J. Tarter, R. Ackermann, W. Barott, et al., The first SETI observations with the Allen Telescope Array, Acta Astronaut. 68 (3-4)(2011) 340-346.

[38] A. L. Zaitsev, METI: Messaging to ExtraTerrestrial Intelligence, Searching for Extraterrestrial Intelligence, The Frontiers Collection. ISBN 9783-642-13195-0. Springer-Verlag Berlin Heidelberg (2011) 399-428

[39] Ambassador for Earth. Is it time for SETI to reach out to the stars? Nature 443 (2006) 606.

[40] http://dsc.discovery.com/tv-shows/other-shows/videos/stephen-hawkings-universe-fear-the-al 


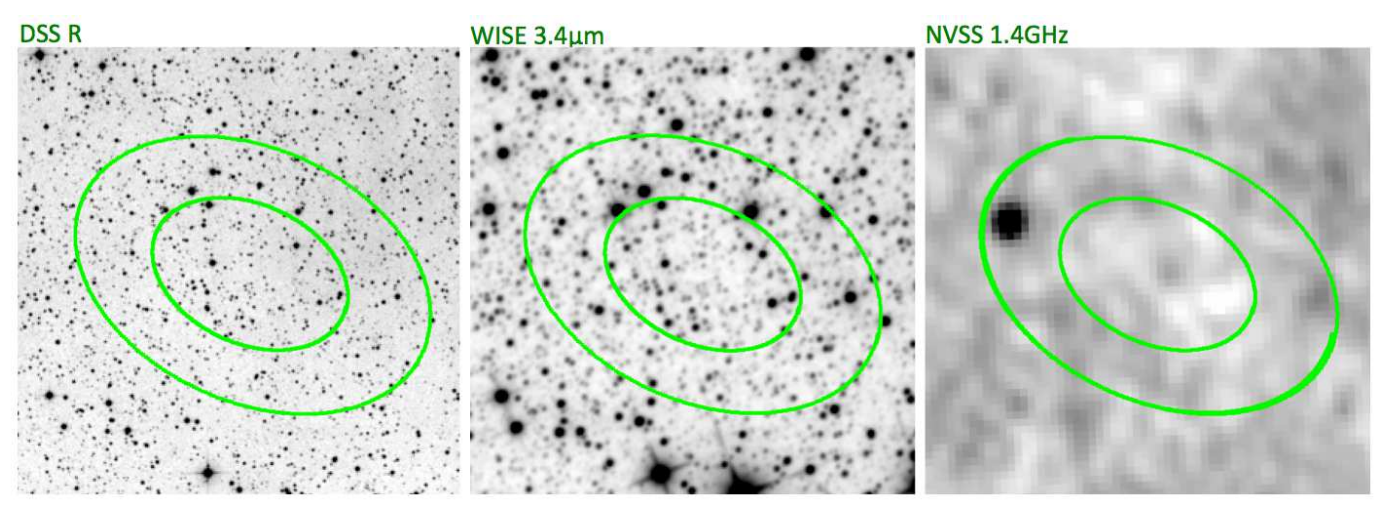

Figure 1: DSS R-band (left), WISE-1 (middle) and NVSS (right) $15^{\prime} \times 15^{\prime}$ image centered on the equatorial coordinates opposite to the ones of Alpha Cen. For each image, the parallactic ellipse of the envisioned ICD is shown for distance to the Sun of $550 \mathrm{AU}$ (outer) and $1000 \mathrm{AU}$ (inner).

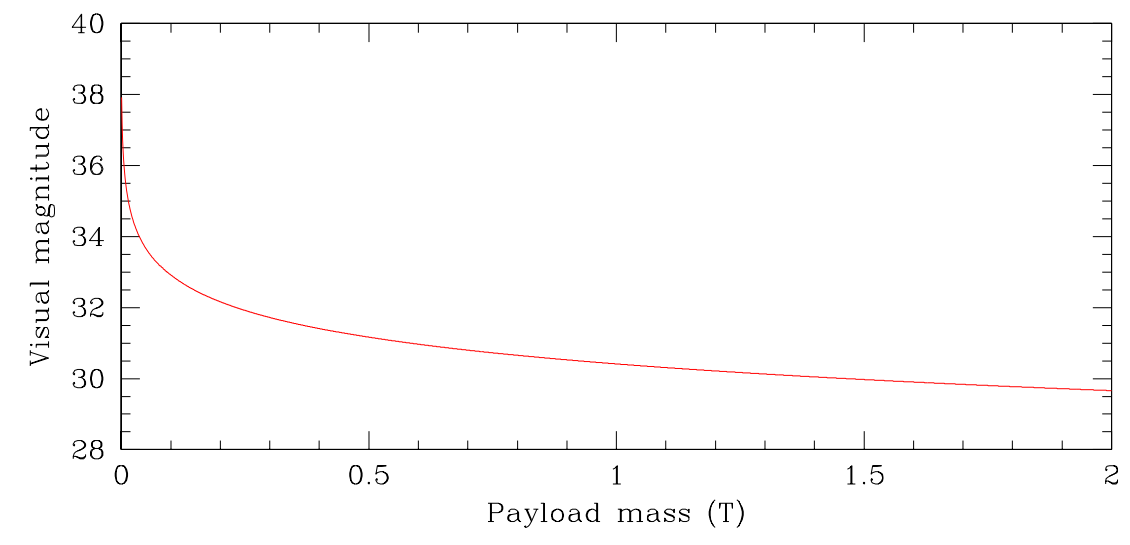

Figure 2: Estimation of the optical magnitude of the ICD as a function of the payload mass. An ideal plane sail observed at zero phase angle is assumed. 